\title{
Characterization of Four Novel H5N6 Avian Influenza Viruses with the Internal Genes from H5N1 and H9N2 Viruses and Experimental Challenge of Chickens Vaccinated with Current Commercially Available H5 Vaccines
}

Peng Chen ${ }^{1}$, Pengwei Zhao ${ }^{2}$, Jun $\mathrm{Chu}^{3}$, Junda Zhu ${ }^{4}$, Qiuchen $\mathrm{Li}^{5}$, Haiyuan Zhao ${ }^{3}$, Yujie $\mathrm{Li}^{3}$, Lingxiang Xin ${ }^{3}$, Xiaoyue Yang ${ }^{3}$, Shijie $\mathrm{Xie}^{3}$, Changdong Zhu ${ }^{3}$, wenbao $\mathrm{Qi}^{3}$, Guanlong $\mathrm{Xu}^{6}$, and Jinxiang $\mathrm{Li}^{7}$

${ }^{1}$ Laboratory of Viral Diseases, National Institute of Allergy and Infectious Diseases, National Institutes of Health, Bethesda, MD 20892

${ }^{2}$ Department of Biochemistry and Molecular Biology, School of Medicine, Zhejiang University

${ }^{3}$ Affiliation not available ${ }^{4}$ College of Veterinary Medicine, China Agricultural University, Beijing 100193, China

${ }^{5}$ Key Laboratory of Animal Epidemiology and Zoonosis, Ministry of Agriculture, College of Veterinary Medicine, China Agricultural University, Beijing 100193, China

${ }^{6}$ China Institute of Veterinary Drug Control

${ }^{7}$ National Agricultural Science and Technology Center

November 18, 2020

\begin{abstract}
Since 2014, highly pathogenic avian influenza H5N6 viruses have been responsible for outbreaks in poultry. In this study, four H5N6 virus strains were isolated from fecal samples of sick white ducks and dead chickens in Shandong in 2019. These H5N6 viruses were triple-reassortant viruses that have not been previously characterized. Their HA genes were derived from the H5 viruses and were closely related to the vaccine strain Re-11. Their NA genes all fell into the N6-like lineage and the internal gene were derived from H5N1 and H9N2 viruses. They all showed high pathogenicity in mice and caused lethal infection with high rates of transmission in chickens. Moreover, the SPF chickens inoculated with the current used vaccine in China were completely protected from these four H5N6 viruses. Our study indicated the necessity of continued surveillance for H5 IAV and the importance of timely update of vaccine strains in poultry industry.
\end{abstract}

\section{Hosted file}

manuscript.pdf available at https://authorea.com/users/376889/articles/493719characterization-of-four-novel-h5n6-avian-influenza-viruses-with-the-internal-genesfrom-h5n1-and-h9n2-viruses-and-experimental-challenge-of-chickens-vaccinated-withcurrent-commercially-available-h5-vaccines 


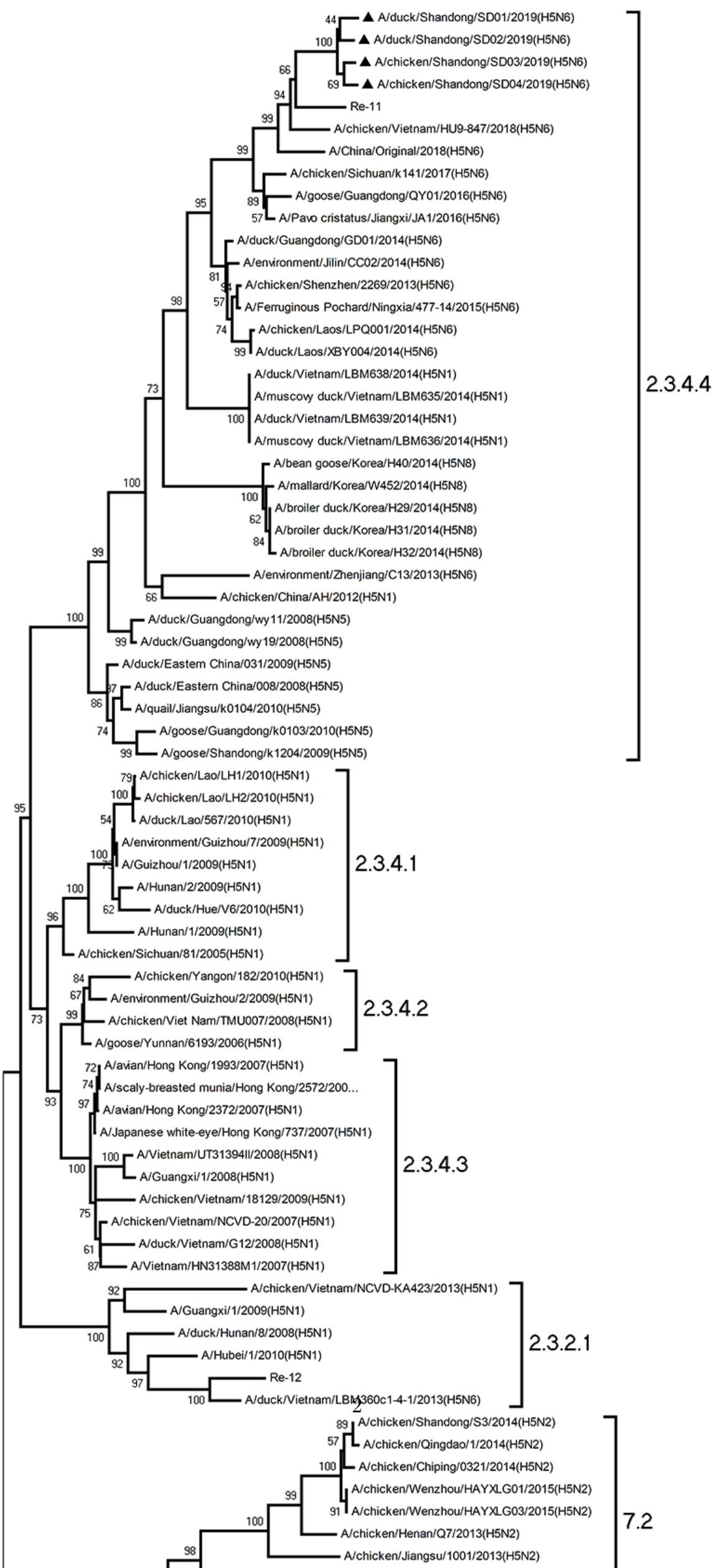




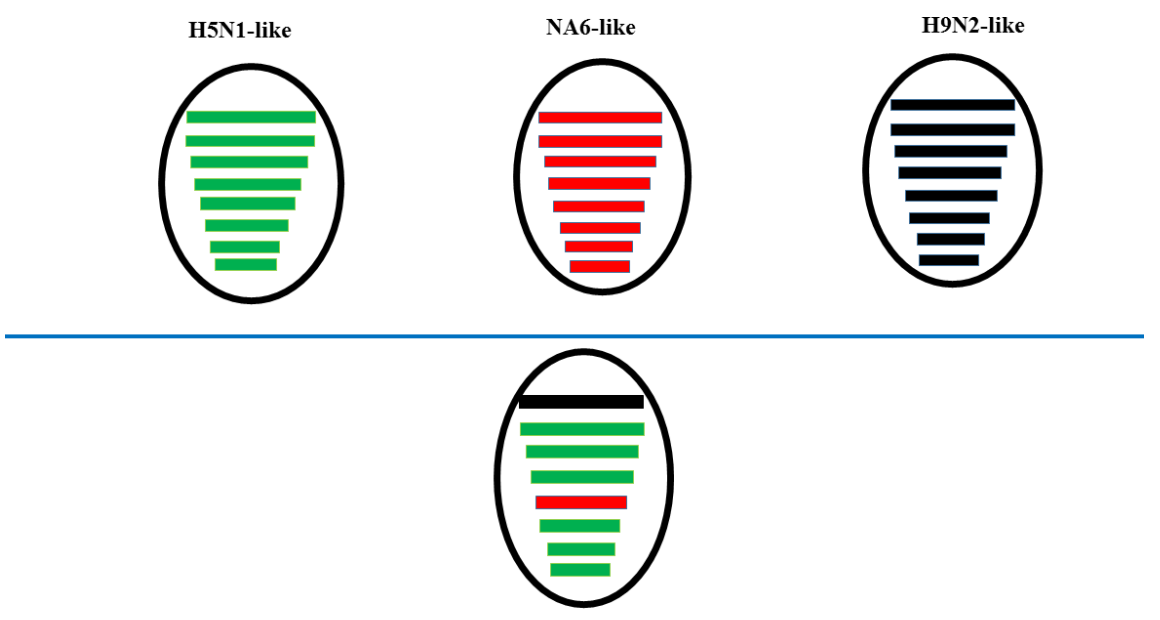

SD01 (H5N6), SD02 (H5N6), SD03 (H5N6), SD04 (H5N6)

Figure 3

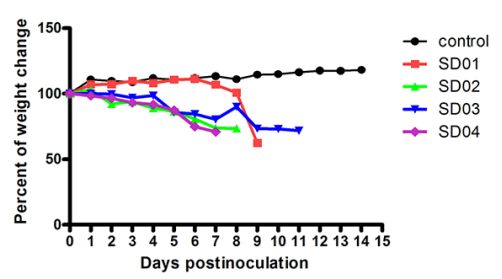

C

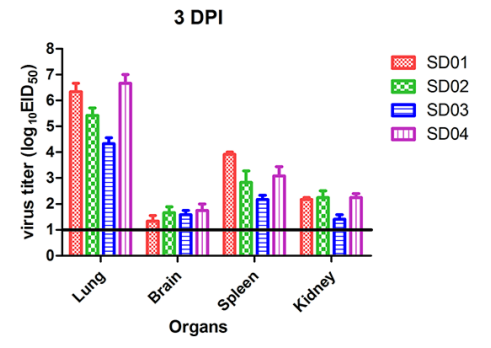

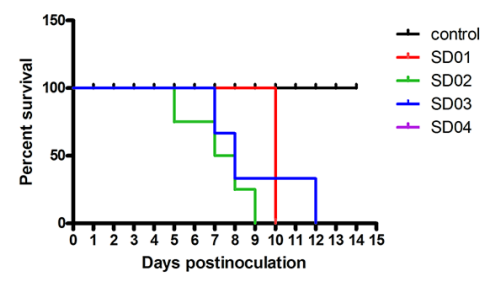

D

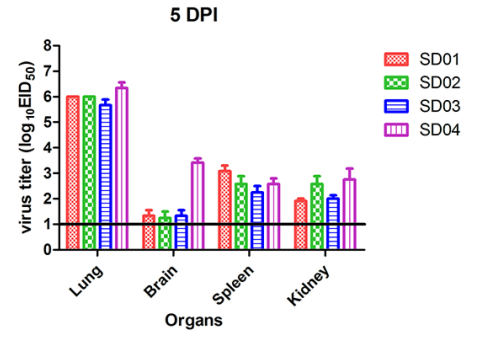


Figure 4

A

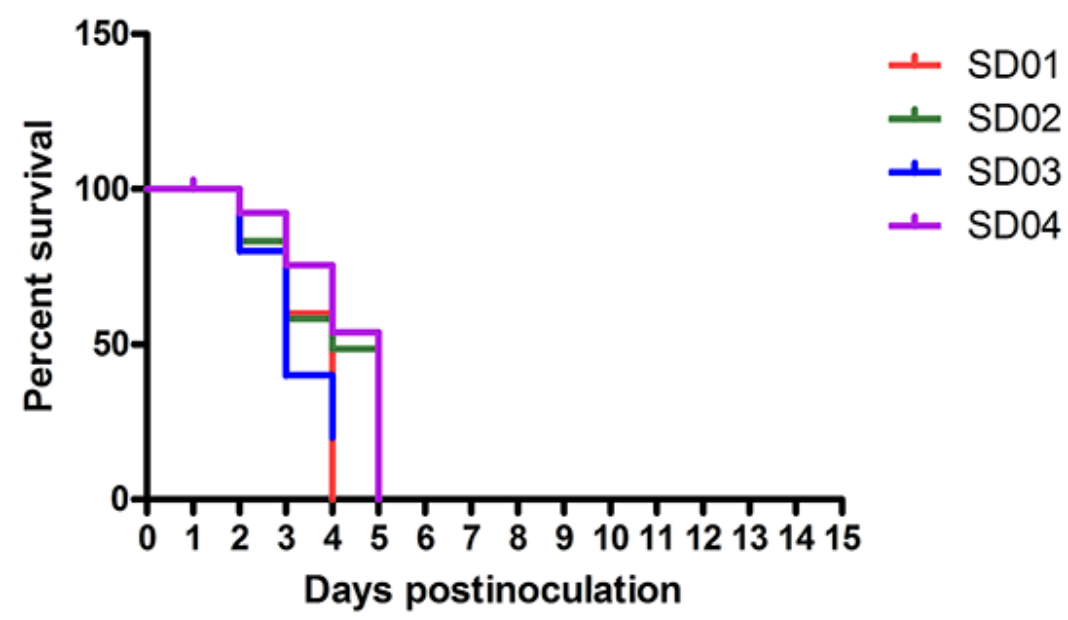

B

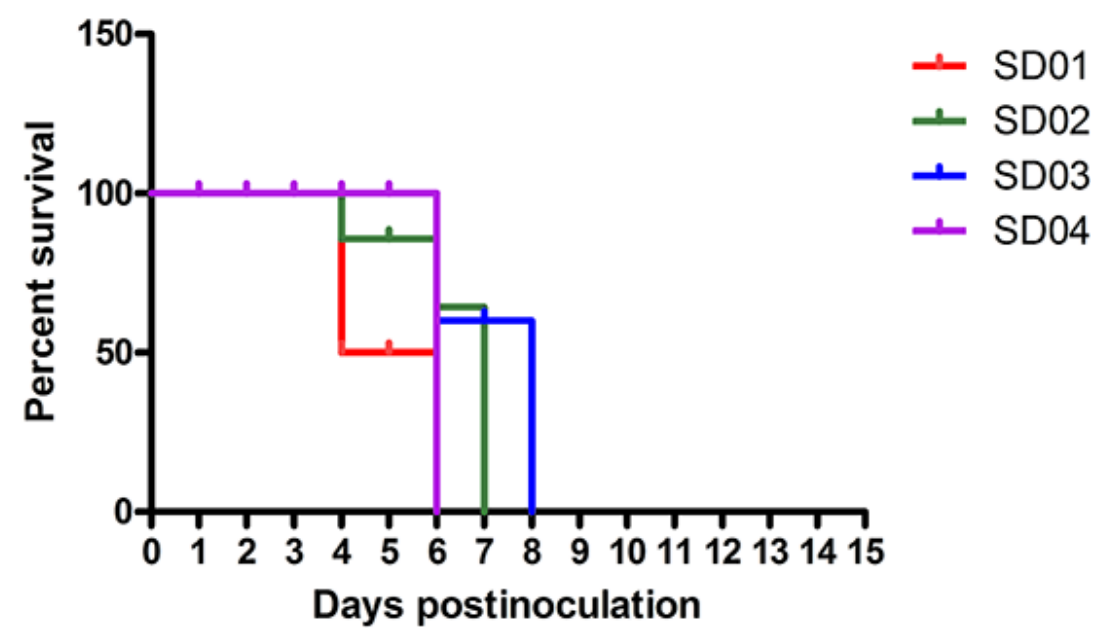




\section{8 days post-immunization}

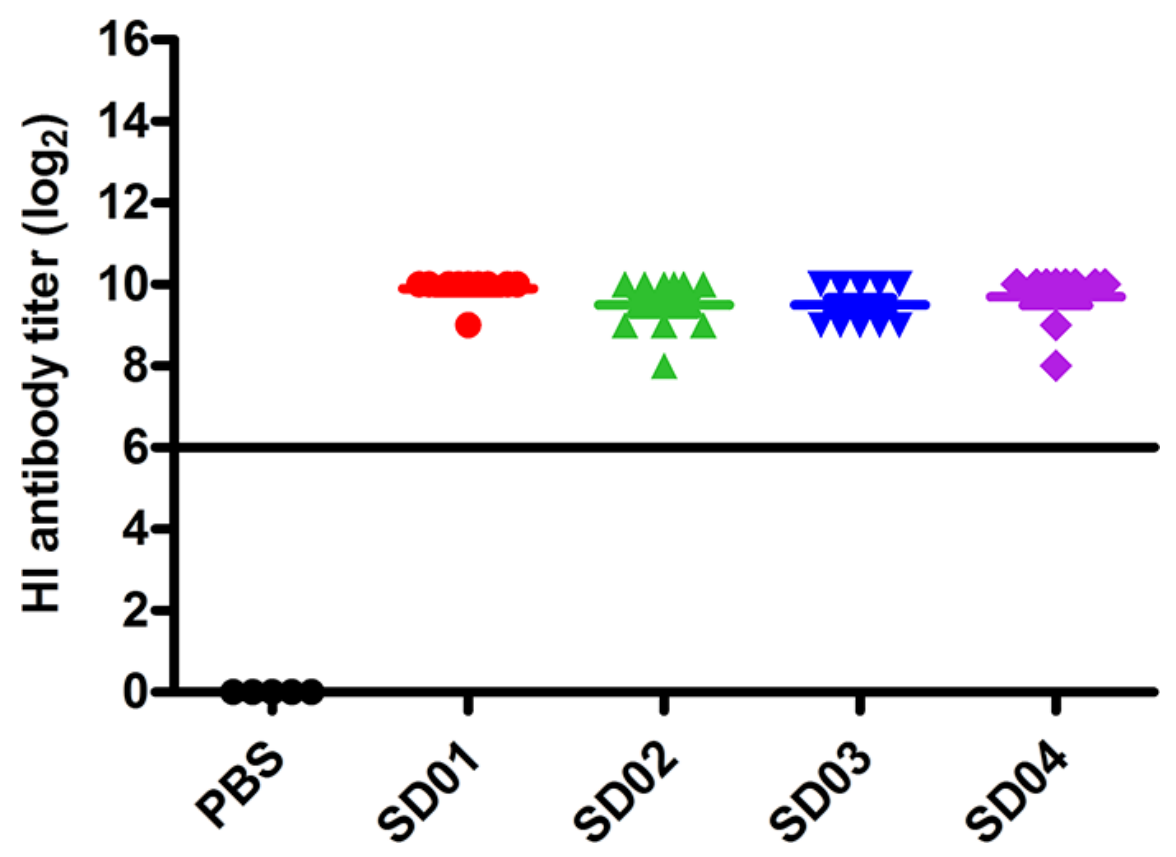

